\title{
Face Recognition Algorithm based on Doubly Truncated Gaussian Mixture Model using DCT Coefficients
}

\author{
D. Haritha \\ Assistant.Professor in CSE \\ Department \\ University College of \\ Engineering, JNTU, Kakinada
}

\author{
K. Srinivasa Rao \\ Professor in Department of \\ Statistics \\ Andhra University \\ Visakhapatnam
}

\author{
Ch. Satyanaraya \\ Associate Professor in CSE \\ Department \\ University College of \\ Engineering, JNTU, Kakinada
}

\begin{abstract}
In this paper a novel and the new method for face recognition is developed and analyzed using doubly truncated multivariate Gaussian mixture model. The 2D DCT coefficients as the feature vector of the each individual face is modelled by $\mathrm{k}$ component mixture of doubly truncated multivariate Gaussian distribution. The number of components and initialization of the model parameter's are obtained by the k-means algorithm and face image histogram. Using the EM algorithm the model parameter's are obtained. A face recognition algorithm is developed by a maximum likelihood function under baysian framework. The efficiency of the developed algorithm is evaluated by obtaining the recognition rates using JNTUK face database and YALE database. This algorithm out perform the face recognition algorithm based on GMM with DCT coefficients.
\end{abstract}

\section{Keywords}

Face recognition, EM algorithm, doubly truncated Gaussian mixture model, DCT coefficients, K-means algorithm.

\section{INTRODUCTION}

A face recognition system is developed to recognize the face of a person from a group of faces. It is a biometric authentication which is used for several security considerations. These face recognition systems are to be classified into two groups namely, closed systems and open systems. In closed systems the claimant face for recognition is considered only from the group of faces stored in the database of fixed size. That is, no outsider will attempt for recognition. In open system the persons from the outside the group may also allowed to claimant the face which is not there in the database. This type of claimant is called imposter. In general closed systems are more useful for document securities and other office related security systems. These closed systems are less complicated than open systems with respect to computational time and logical considerations $[23,24]$. The basic applications of the face recognition system are designing the access control systems for devices like personal mobile devices (PMD) building gate control, etc,.

Different researchers have developed various face recognition systems for the decision maker to accept or reject a claim depending on a score like likelihood ratio, distance measure, MLP, etc, using a threshold. Over the last few years several improvements have been taken care in face recognition systems. Kieron Messer et al., (2004) [15] has given comparative study of different face recognition systems and categorized them into two groups namely discriminative approaches and generative approaches.

In discriminative approaches a binary decision regarding the acceptance or rejection is considered by utilizing the macro information of the face. That is why these approaches are known as holistic approaches. In which the pixel intensity of the gray scale face image is converted into some feature vector's. In this method the prinicipal component subspace is using principal component analysis or eigen faces is adopted for face recognition [2,7,16,20,25,26,28]. The linear discriminant subspace known as LDA or fisher faces are used for face recognition system $[4,12,17,27]$. Illumination invariant features such as histogram equalization or LBP are used for face recognition systems [1,30].

In general, generative approaches are more efficient than discriminative approaches. Hence, the face recognition systems are developed using Gaussian mixture models $[5,18,22]$. These approaches are more robust and effective for automatic face recognition systems, since they adopt model based considerations.

In all these model based face recognition systems the feature vector is characterized through a probability distribution. The important step in this method is feature vector extraction. Several types of methods are adopted for extracting the feature vector of a face image. Among them 2D Discrete Cosine transformation or DCTmod2 or 2D Gabor Wavelets are more important $[10,13,14]$. In all these methods it is customary to consider that the DCT coefficients (feature vector) follows a Gaussian mixture model. The Gaussian mixture model has certain drawbacks such as the feature vector in each component is miso kurtic and having infinite range. But in many face recognition problems the feature vector (DCT coefficients) may not be distributed as miso kurtic and symmetric. In addition to this, all DCT coefficients of the face image are having finite range. Hence ignoring the reality of finite range with asymmetric nature of the feature vector may bring falsification in the face recognition models and may not be accurate.

Hence in this paper we develop and analyze a face recognition model based on Doubly truncated multivariate GMM. The doubly truncated Gaussian mixture model is capable of portraying several probability distributions like asymmetric / symmetric / platy kurtic / lepty kurtic distributions [19,21]. Various approaches are discussed by different researchers on the problem of face recognition. But, there is no serious work is done on face recognition with doubly truncated GMM. So, we propose a generative 
approach for face recognition, based on doubly truncated GMM. We used K-means algorithm for determining the number of components in each face and to obtain initial estimates of the model parameters. This model also includes GMM as a limiting base. The performance of the model was compared with the GMM.

The paper is structured as follows. Section 2 summarizes feature extraction, Section 3 summarizes truncated Gaussian mixture face recognition model, Section 4 summarizes the estimation of parameters using EM Algorithm, Section 5 summarizes initialization of model parameters and Section 6 summarizes the face recognition algorithm, experimental results are given in Section 7, and finally, conclusions are presented in Section 8.

\section{FEATURE EXTRACTION}

For developing the face recognition model, the important consideration is deriving the features of the each individual face image. Several techniques are adopted to extract the feature vector associated with the each individual face [10]. Among the transformation used for feature vector extraction the 2D Discrete Cosine Transform is simple and more efficient in characterizing the face of the individual. This method has been recognized as world wide standard [JPEG] for image compression [3].

For obtaining the feature vector associated with the each individual face we assume that it consists of $\left(N_{P} \times N_{P}\right)$ blocks. In each block the 2D DCT coefficients are computed using the method [10]. The computation of 2D DCT coefficients for a face of $\left(\mathrm{N}_{P} \times \mathrm{N}_{P}\right)$ blocks is given in $\mathrm{x}_{\mathrm{i}}$.

These coefficients are ordered according to a zig-zag pattern (consisting of 16 coefficients) reflecting the amount of information stored as given in [13]. After comprehensing the DCT coefficients we get the feature vector of the each individual face as $\mathrm{x}_{\mathrm{i}}=\left[\begin{array}{lllll}c_{1} & c_{2} & \cdots & c_{M}\end{array}\right]^{\mathrm{T}}$ consisting of $\mathrm{N}_{\mathrm{P}} \mathrm{x} 15$ coefficients.

\section{DOUBLY TRUNCATED GAUSSIAN MIXTURE FACE RECOGNITION MODEL}

In this section we briefly discuss the probability distribution (model) used for characterizing the feature vector of the face recognition system. After extracting the feature vector of each individual face it can be modelled by a suitable probability distribution such that the characteristics of the feature vector should match the statistical theoretical characteristics of the distribution. Since each face is a collection of several components like mouth, eyes, nose, etc, the feature vector characterizing the face is to follow a M-component mixture distribution. In each component the feature vector is having finite range it can be assumed to follow a doubly truncated Gaussian distribution. This in turn implies that the feature vector of each individual face can be characterized by a Mcomponent doubly truncated Gaussian mixture model. The joint probability density function of the feature vector associated with each individual face is

$$
h((\vec{x} \mid \lambda))=\sum_{i=1}^{M} \alpha_{i} d_{i}(\vec{x})
$$

where, $d_{i}(\vec{x})$ is the probability density function of the ith component feature vector which is of the form doubly truncated Gaussian distribution [23]

$$
\begin{gathered}
d_{i}(\vec{x})=\left(\frac{1}{(B-A)(2 \pi)^{\frac{D}{2}}\left|\Sigma_{i}\right|^{\frac{1}{2}}}\right) \\
* \exp \left\{-\frac{1}{2}\left(\overrightarrow{x_{i}}-\overrightarrow{\mu_{i}}\right)^{\prime} \Sigma_{i}^{-1}\left(\overrightarrow{x_{i}}\right.\right. \\
\left.\left.-\overrightarrow{\mu_{i}}\right)\right\}
\end{gathered}
$$

where, $\vec{x}$ is a $\mathrm{D}$ dimensional random vector $\left(\overrightarrow{x_{t}}=\right.$ $\left.\left(x_{1} x_{2} \ldots x_{t}\right)\right)$ is the feature vector, $\overrightarrow{\mu_{i}}$ is the $\mathrm{i}^{\text {th }}$ component feature mean vector, $\Sigma_{i}$ is the $\mathrm{i}^{\text {th }}$ component of co-variance matrix,

$A=\int_{-\infty}^{x_{\mathrm{L}}} \cdots \int_{-\infty}^{x_{L}} d_{i}\left(\overrightarrow{x_{t}}\right) \vec{d} x_{t}$ and

$B=\int_{-\infty}^{\mathrm{x}_{\mathrm{M}}} \ldots \int_{-\infty}^{x_{M}} d_{i}\left(\overrightarrow{x_{t}}\right) \vec{d} x_{t}$.

The mean vector of the component feature is

$E(\vec{x})=\mu_{i}+\sigma_{i}^{2}\left[\frac{f\left(\overrightarrow{\mathrm{x}_{\mathrm{L}}}\right)-f\left(\overrightarrow{\mathrm{x}_{\mathrm{M}}}\right)}{\emptyset\left(\overrightarrow{\mathrm{x}_{\mathrm{L}}}\right)-\emptyset\left(\overrightarrow{\mathrm{x}_{\mathrm{M}}}\right)}\right]$

where, $\varnothing\left(\overrightarrow{\mathrm{x}_{\mathrm{L}}}\right)$ and $\emptyset\left(\overrightarrow{\mathrm{x}_{\mathrm{M}}}\right)$ are the standard normal areas and $\overrightarrow{\mathrm{x}_{\mathrm{L}}}, \overrightarrow{\mathrm{x}_{\mathrm{M}}}$ are the lower and upper truncated points of the feature vectors. $d_{i}(\vec{x}), i=1 \ldots . M$ are the component densities and $\alpha_{i}(\vec{x}), i=1 \ldots . M$ are the mixture weights, with mean vector. The mixture weights satisfy the constraints $\sum_{i=1}^{M} \alpha_{i}=1$

The variance of each feature vector is $\Sigma$ with diagonal elements as

$V_{i}^{2}=\left[1+\frac{\left(\frac{\overrightarrow{\mathrm{x}_{\mathrm{L}}}-\overrightarrow{\mu_{i}}}{\sigma_{i}}\right) \overrightarrow{\mathrm{x}_{\mathrm{L}}}-\left(\frac{\overrightarrow{\mathrm{x}_{\mathrm{M}}}-\overrightarrow{\mu_{i}}}{\sigma_{i}}\right) \overrightarrow{\mathrm{x}_{\mathrm{M}}}}{B-A}\right]$

The DTGMM is parameterized by the mean vector, Co-variance matrix and mixture weights from all components densities. The parameters are collectively represented by the parameter. Set $\lambda_{i}=\left\{\alpha_{i}, \mu_{i}, \Sigma_{i}\right\} i=1,2, \ldots M$. For face recognition each image is represented by it's model parameters.

The doubly truncated multivariate Gaussian mixture model can represent different forms depending on the choice of the co-variance matrix for all Gaussian component(Grand co-variance) or a single co-variance matrix shared by all face models(global covariance) used in DTGMM. The covariance matrix can also be full or diagonal. Here, we used diagonal covariance matrix for our face model. This choice is based on the works given by [11] and initial experimental results indicating better identification performance and hence $\Sigma$ can be represented as

$$
\Sigma=\left[\begin{array}{cccc}
V_{1}{ }^{2} & 0 & 0 & 0 \\
0 & V_{2}^{2} & 0 & 0 \\
- & - & - & - \\
- & - & - & - \\
0 & 0 & 0 & V_{M}^{2}
\end{array}\right]
$$

This simplifies the computational complexities. The doubly truncated multivariate Gaussian mixture model includes the GMM model as a particular case when the truncation points tends to infinite. 


\section{ESTIMATION OF THE MODEL PARAMETERS}

For developing the face recognition model it is needed to estimate the parameters of the face model. For estimating the parameters in the model we consider the EM algorithm which maximizes the likelihood function of the model for a sequence of $i$ training vectors $\left(\overrightarrow{x_{t}}=\left(x_{1} x_{2} \ldots x_{t}\right)\right)$.

The likelihood function of the sample observations is

$$
L\left(\vec{x} ; \lambda_{j}\right)=\prod_{i=1}^{T} h\left(\vec{x} ; \lambda_{j}\right)
$$

where, $h\left(\vec{x} ; \lambda_{j}\right)$ is given in equation (1).

The likelihood function contains the number of components $\mathrm{M}$ which can be determined from the k-means algorithm given by sailaja et al (21). The k-means algorithm requires the initial number of components which can be taken by plotting the histogram of the face image using MATLAB code and counting the number of peaks. Once M-is assigned the EM algorithm can be applied for refining the parameters. The updated equations of the parameters of the model are:

$$
\begin{gathered}
\alpha_{k}^{l+1}=\frac{1}{T} \sum_{i=1}^{T} h\left(i \mid \overrightarrow{x_{t}}, \lambda_{j}\right) \\
\mu_{k}^{l+1}=\frac{\sum_{i=1}^{T} \overrightarrow{x_{t}} h\left(i \mid \overrightarrow{x_{t}}, \lambda_{j}\right)+\sum_{i=1}^{T} \frac{f\left(\overrightarrow{x_{\mathrm{M}}}\right)-f\left(\overrightarrow{x_{\mathrm{L}}}\right)}{B-A} \sigma_{k}{ }^{2} h\left(i \mid \overrightarrow{x_{t}}, \lambda_{j}\right)}{\sum_{i=1}^{T} h\left(i \mid \overrightarrow{x_{t}}, \lambda_{j}\right)} \\
\sigma_{k}^{l+1}=\frac{\sum_{i=1}^{T} h\left(i \mid \overrightarrow{x_{t}}, \lambda_{j}\right)\left(\overrightarrow{x_{t}}-\mu_{i}^{l+1}\right)}{c \sum_{i=1}^{T} h\left(i \mid \overrightarrow{x_{t}}, \lambda_{j}\right)}
\end{gathered}
$$

where,

$$
c=\frac{1}{B-A}\left(1+\mu_{k}^{l+1}\right)\left[\left(f\left(\overrightarrow{\mathrm{x}_{\mathrm{M}}}\right)-f\left(\overrightarrow{\mathrm{x}_{\mathrm{L}}}\right)\right)+\right.
$$$$
\left.\left(\mathrm{x}_{\mathrm{M}} f\left(\overrightarrow{\mathrm{x}_{\mathrm{M}}}\right)-\mathrm{x}_{\mathrm{L}} f\left(\overrightarrow{\mathrm{x}_{\mathrm{L}}}\right)\right)\right] \text {, }
$$

$$
f\left(\mathrm{x}_{\mathrm{M}}\right)=\int_{-\infty}^{\mathrm{x}_{\mathrm{M}}} d_{i}\left(\overrightarrow{x_{t}}\right) \vec{d} x_{t}, f\left(\mathrm{x}_{\mathrm{L}}\right)=\int_{-\infty}^{\mathrm{x}_{\mathrm{L}}} d_{i}\left(\overrightarrow{x_{t}}\right) \vec{d} x_{t}
$$
and

$$
h\left(i \mid \overrightarrow{x_{t}}, \lambda_{j}\right)=\frac{\alpha_{i} d_{i}\left(\overrightarrow{x_{t}}\right)}{\sum_{i=1}^{k} \alpha_{i} d_{i}\left(\overrightarrow{x_{t}}\right)}
$$

\section{INITIALIZATION OF MODEL PARAMETERS}

To utilize the EM algorithm we have to initialize the parameters $\left\{\alpha_{i}, \mu_{i}, \sigma_{i}\right\}, i=\{1 \ldots . M\} . \mathrm{X}_{\mathrm{M}}$ and $\mathrm{X}_{\mathrm{L}}$ are estimated with the maximum and the minimum values of each feature respectively. The initial values of $\alpha_{i}$ can be taken as $\alpha_{i}=\frac{1}{M}$. The initial estimates of $\alpha_{i}, \mu_{i}$ and $\sigma_{i}$ of the $\mathrm{i}^{\text {th }}$ component are obtained by using the method given by A.C.Cohen[8].

\section{FACE RECOGNITION SYSTEM}

Face Recognition means recognizing the person from a group of $\mathrm{H}$ samples. The figure 1 describes the face recognition algorithm under study.

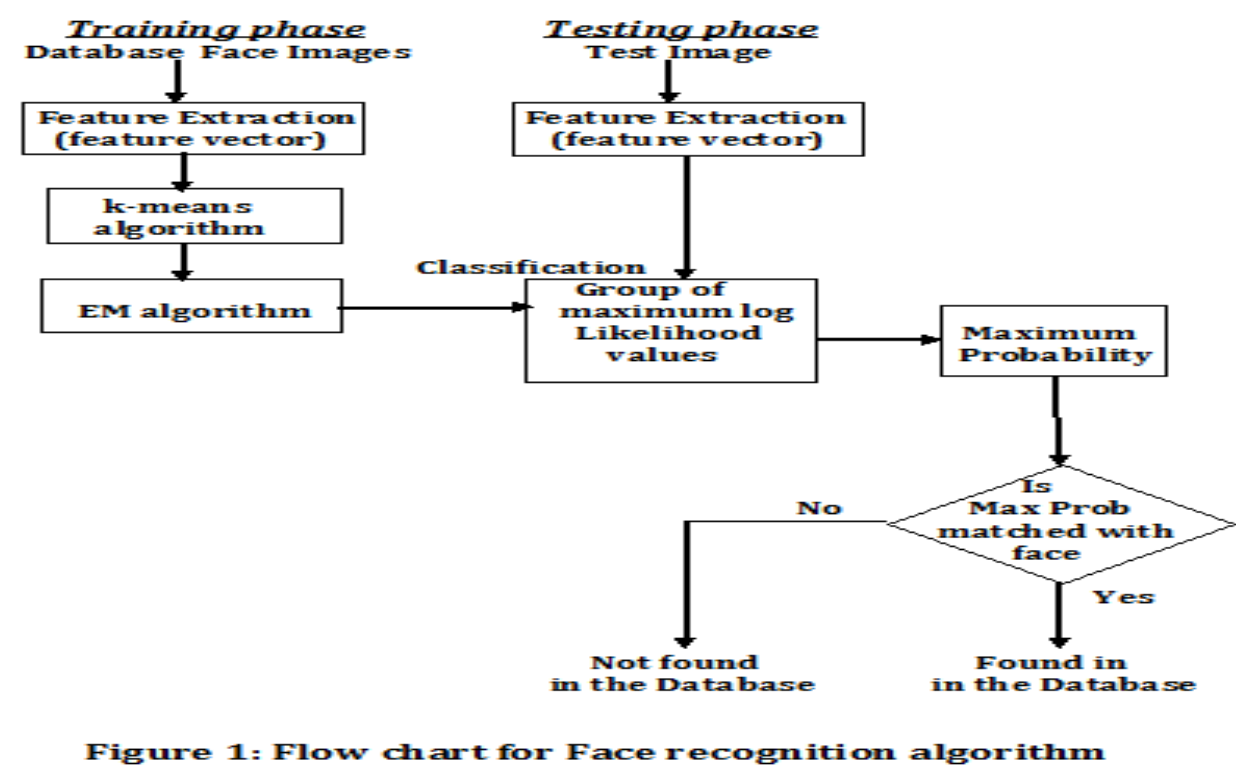

The feature vectors are obtained by using the procedure discussed in section 2. By using $\mathrm{K}$-means algorithm we divide the $\mathrm{T}$ samples into $\mathrm{M}$ groups. We take initially $\alpha_{i}=\frac{1}{M}, i=$ $1,2, \ldots M$ and find means $\left(\mu_{i}\right)$ and variances $\left(V_{i}\right)$ of each feature for each group. Using the EM algorithm the refined estimates of the model parameters $\left\{\alpha_{i}, \mu_{i}\right.$ and $\left.\sigma_{i}\right\}$ for each component of each face Image are obtained. For the given a claim of a person C's identity and a set of feature vectors $\mathrm{X}$, supporting the claim, the log likelihood of the claimant is calculated using

$$
L\left(X \mid \lambda_{C}\right)=\frac{1}{T} \sum_{i=1}^{T} \log h\left(\overrightarrow{x_{i}} \mid \lambda_{C}\right)
$$

where, $\mathrm{h}(x \mid \lambda)$ which is given in eq (1).

Find the log likelihood value for all faces in the training group of samples, each represented by DTGMM's with parameters $\left(\lambda_{1}, \lambda_{2}, \ldots . \lambda_{H}\right)$. The face which has the maximum posterior probability for a given, feature vector that the face is matched with the person, i.e., the $\mathrm{k}^{\text {th }}$ face for which log likelihood is $\operatorname{maximum}\left(\widehat{H}=\arg \max _{1<k<H} \sum_{i=1}^{T} \log h_{r}\left(\overrightarrow{x_{t}} \mid \lambda_{k}\right)\right)$ out of all faces. 


\section{EXPERIMENTAL RESULTS}

The performance of the developed algorithm is evaluated using two types of databases namely JNTUK and yale face databases [23,29,4]. Sample of 20 persons images from JNTUK database is shown in figure. 2 .

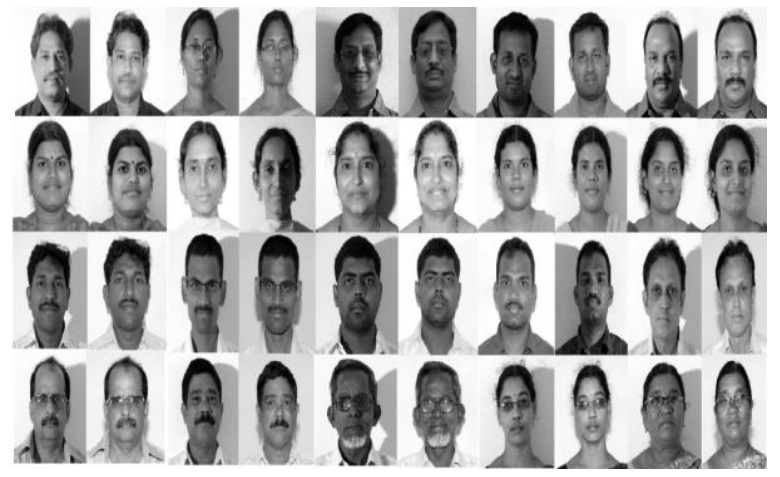

Figure 2: Sample Images form JNTUK database

The whole dataset are divided into test and training datasets. The training faces were first processed by feature vector extraction explained in section 2 to produce the transformed feature vector (DCT coefficients) for each face. For each face the image histogram is drawn and the number of components $\mathrm{M}$ is initially obtained by counting the number of peaks in each histogram. The histogram of the sample face image of a person is shown in figure.3. The k-means algorithm is utilized for dividing the samples of the each image features into $\mathrm{M}$ components like face, chick, nose, forehead, etc. Using these $\mathrm{M}$ groups the model parameter's in each component are initially estimated with the method given by A.C.Cohen [8]. With these initial coefficients and the updated equations of the EM algorithm the model parameters of the doubly truncated multivariate Gaussian mixture model of each face is estimated. With the test dataset the face recognition algorithm is validated by computing the recognition rate with its confidence limits. Table. 1 shows the recognition rate of the face recognition system using GMM and DTGMM.

Table 1: Face recognition rates

\begin{tabular}{|c|c|c|}
\hline \multirow{2}{*}{ Database } & \multicolumn{2}{|c|}{ Recognition rate } \\
\cline { 2 - 3 } & GMM & DTGMM \\
\hline JNTUK & $89.5 \pm 1.5$ & $96.17 \pm 1.3$ \\
& & \\
\hline yale & $89.39 \pm 2.1$ & $96.07 \pm 1.2$ \\
& & \\
\hline
\end{tabular}

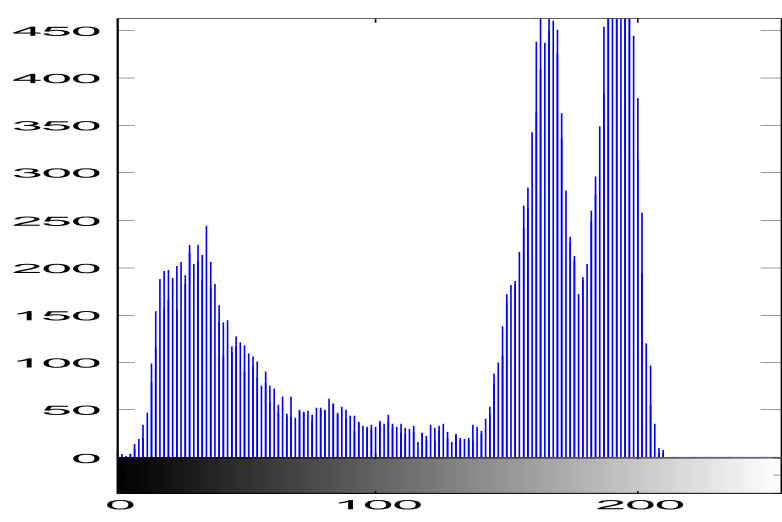

Figure 3: Histogram of the feature vector

From Table. 1 it is observed that the proposed algorithm identifies the $96.17 \%$ and $96.07 \%$ for JNTUK and yale faces correctly. The computation time for face recognition algorithm is 10.55688 seconds for testing and 49.58836 seconds for training in DTGMM for a data set of 120 images. Graph-1 describes the recognition rate for different sizes of datasets. It is observed that the recognition rate stabilized after the data size of 25 faces. i.e. the algorithm is consistent with small and large databases for face recognition.

Since the proposed face recognition system performance is linked with the number of DCT coefficients the optimal number of DCT coefficients required for effective recognition of the system is evaluated. For different number of DCT coefficients taken from the total of 64 coefficients of each block in say $5,10,15,20,25,30,35$ and 40 the recognition rates are of the system computed for both the proposed model and the model with GMM. Table. 2 shows the computed recognition rates for both JNTUK and yale databases using the face recognition system based on DTGMM and GMM. Gaph.2 shows the relationship between recognition rates and number of DCT coefficients.

Table 2: Recognition rates for different number of DCT Coefficients.

\begin{tabular}{|c|c|c|c|c|}
\hline \multirow{3}{*}{$\begin{array}{l}\text { Number of } \\
\text { DCT } \\
\text { coefficients }\end{array}$} & \multicolumn{4}{|c|}{ Recognition rate } \\
\hline & \multicolumn{2}{|c|}{ JNTUK } & \multicolumn{2}{|c|}{ yale } \\
\hline & GMM & DTGMM & GMM & DTGMM \\
\hline 5 & 50.63 & $60.23 \pm 1.2$ & 50.34 & $60.18 \pm 1.1$ \\
\hline 10 & 70.12 & $82.4 \pm 1.1$ & 70.04 & $82.3 \pm 1.4$ \\
\hline 15 & 89.5 & $96.17 \pm 1.3$ & 89.39 & $96.07 \pm 1.2$ \\
\hline 20 & 85.1 & $91.5 \pm 1.2$ & 85.06 & $91.4 \pm 1.1$ \\
\hline 25 & 82.68 & $91.5 \pm 1.3$ & 82.61 & $91.4 \pm 1.1$ \\
\hline 30 & 82.68 & $91.5 \pm 1.1$ & 82.61 & $91.4 \pm 1.3$ \\
\hline 35 & 82.68 & $91.5 \pm 1.2$ & 82.61 & $91.4 \pm 1.3$ \\
\hline 40 & 82.68 & $91.5 \pm 1.3$ & 82.61 & $91.4 \pm 1.1$ \\
\hline
\end{tabular}




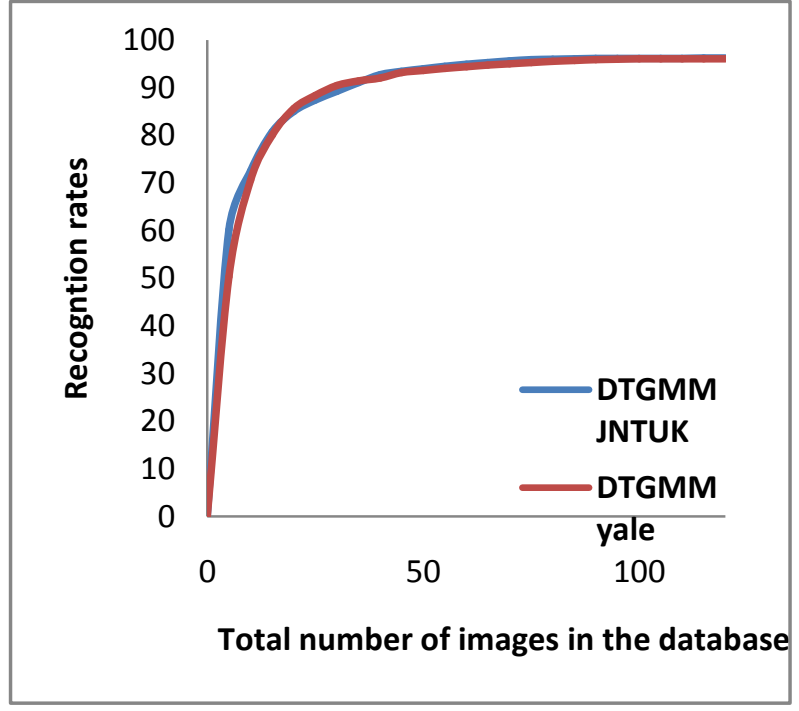

Graph 1: Recognition rates of different databases

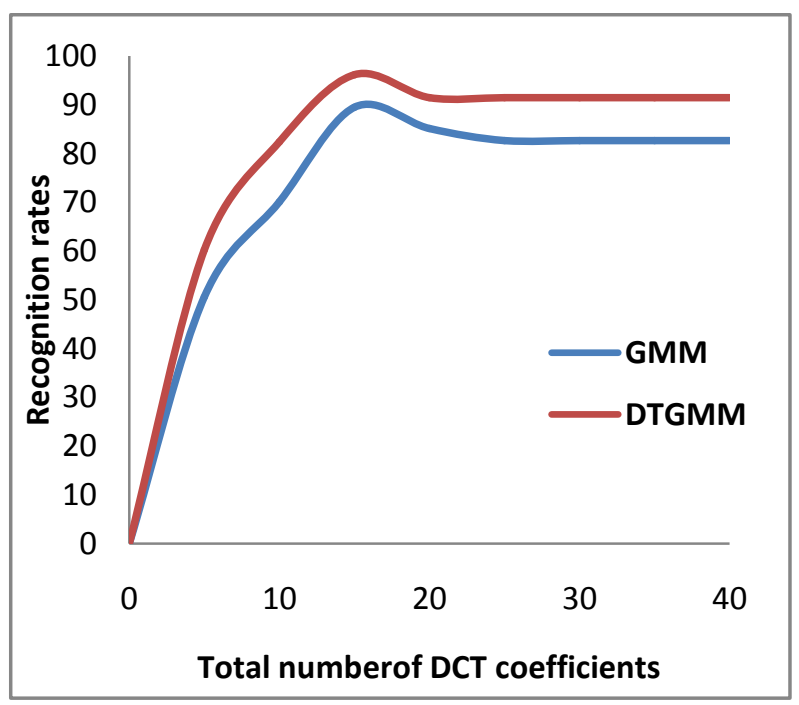

\section{Graph 2: Recognition rates versus toal number of} DCT coefficients on JNTUK dataase

From Table.2 and Graph.2, it is observed that for both JNTUK and yale databases the recognition rate stabilizes after 15 DCT coefficients for the face recognition system with DTGMM. Whereas, the recognition rate stabilizes after 20 DCT coefficients for the face recognition system with GMM. This indicates even with low dimension feature vector also will have high recognition rate with DTGMM model. This feature has a significant effect on execution time of the system.

\section{CONCLUSION}

In this paper we proposed the face recognition model based on doubly truncated GMM with K-means algorithm. The model parameters are estimated through EM algorithm. After identifying the number of components densities for each face we developed a global model for face recognition. This algorithm is evaluated using JNTUK and Yale databases. The experimental results shows that the face recognition model using DTGMM has better recognition rate compared to the face recognition model with GMM. This algorithm is useful for authentication and surveillance. The DCT coefficients model for face recognition algorithm simplifies the computations and utilizes the overall information of the face image. This method can also extended to HMM with DTGMM which will be considered in future work.

\section{REFERENCES}

[1] Ahonen T., Hadid A., and Pietik"ainen M. (2004), “ Face recognition with local binary patterns", in Proc. 8th European Conference on Computer Vision (ECCV), Prague, Czech Republic, pp.469-481.

[2] Akamatsu S., Sasaki T., Fukamachi H., and Suenaga Y. (1991), "A robust face identification scheme- KL expansion of an invariant feature space", in SPIE Proceedings of Intell. Robots and Computer Vision X. Algorithms and Techn., 1607, pp.71-84.

[3] Annadurai S., Saradha A., (2004),“ Discrete Cosine Transform based face recognition using Linear Discriminant Analysis “, Proceedings of International Conference on Intelligent Knowledge Systems(IKS2004).

[4] Belhumeur P., Hespanha J. P., and Kriegman D. J. (1996), "Eigenfaces vs. Fisherfaces: Recognition using class specific linear projection”, in ECCV’96, pp.45-58.

[5] Cardinaux F., Sanderson C., Bengio S. (2004), “ Face Verification using Adaptive Generative Models", in Proc. 6th IEEE Int. Conf. Automatic Face and Gesture Recognition (AFGR), pp. 825-830.

[6] Cardinaux F., Sanderson C., and Marcel S. (2003), "Comparison of MLP and GMM classifiers for face verification on XM2VTS", in 4th International Conference on Audio- and Video-Based Biometric Person Recognition (AVBPA), pp. 911-920.

[7] Chellappa R., Wilson C., and Sirohey S. (1995), "Human and machine recognition of faces: A survey", in Proc. IEEE 83(5): pp.705-740.

[8] Cohen A.C. Jr.,(1950), "Estimating the Mean and Variance of Normal Populations from Singly and Doubly Truncated Samples", Ann. Maths. Statist., 21, pp.557569.

[9] Conrad Sanderson, Fabien Cardinaux, Samy Bengio (2005), "On Accuracy/Robustness/ Complexity TradeOffs in Face Verification", in Proceedings of the Third International Conference on Information Technology and Applications (ICITA'05), IEEE.

[10] Conrad Sanderson, Kuldip K. Paliwal (2003), "Fast features for face Recognition under illumination direction changes", in Pattern Recognition Letters Vol 24, No.14, pp.2409-2419.

[11] Douglas A. Reynolds, and Richard C. Rose,(1995), "Robust Text Independent speaker identification using Gaussian Mixture Speaker Model", IEEE Tran. Speech and Audio Processing, 3, pp.72-83.

[12] Duba R.O. and Hart P.E.(1973),"Pattern Classification and Scene Analysis",Wiley, New York.

[13] Gonzales R.C., Woods R.E.,(1992), "Digital Image Processing", Addison -Wesley.

[14] Haritha D. and Satyanarayana Ch.,(2010), “ Performance evaluation of face Recognition using DCT approach", international Conferrence on statistics, 
probability, operations, Research, Computer Science \& allied Areas in conjunction with IISA \& ISPS , pp:86.

[15] Kieron Messer, Josef Kittler, Mohammad Sadeghi, Miroslav Hamouz, Alexey Kostyn, Sebastien Marcel, Samy Bengio, Fabien Cardinaux, Conrad Sanderson, Norman Poh, Yann Rodriguez, Krzysztof Kryszczuk, Jacek Czyz, Vandendorpe L, Johnny Ng, Humphrey Cheung, and Billy Tang, (2004), "Face Recognition competition on the BANCA database", in Proceedings of the International Conference on Biometric Recognition (ICBA), Hong Kong, July, pp.15-17.

[16] Kirby M. and sirvoich L. (1990), “Application of the Karhunen-Loeve procedure for the characterization of human faces", IEEE Trans. On Patt, Anal. And Machine Intell. 12, pp.103-108.

[17] Marcel S. (2004), "A symmetric transformation for LDA-based face verification", in Proceedings of the $6^{\text {th }}$ International Conference on Automatic Face and Gesture Recognition, IEEE Computer Society Press.

[18] Nefian A. and Hayes M. (1999), "Face recognition using an embedded HMM", in Proceedings of the IEEE Conference on Audio and Video-based Biometric Person Recognition (AVBPA), pp .19-24.

[19] Norman L. Johnson Samuel kotz, N. Balakrishnan, (1995), “ Univariate Distributions volume1, second edition, wiley student edition.

[20] Pentland A., Moghaddam B., Starner T., and Turk M. (1994), "View-based and modular eigenspaces for recognition", in proc. IEEE Computer Soc. Conf. On Computer Vision and Patt. Recog., pp.84-91.

[21] Sailaja V., Srinivasa Rao K., and Reddy K.V.V.S.,(2010), “ Text independent Speaker Identification with Doubly Truncated Gaussian Mixture Model", international Journal of Information Tevchnology and Knowledge Management, Volume2, No. 2, pp.475-480.

[22] Samaria F. (1994), "Face Recognition Using Hidden Markov Models", PhD Thesis, University of Cambridge.
[23] Satyanarayana Ch., Haritha D., Sammulal P. and Pratap Reddy L.,(2008), “ updation of face space for face recognition using PCA", proceedings of the international conference on RF \& signal processing system (RSPS08), vol.1, pp. 195-202, vijayawada, INDIA.

[24] Satyanarayana Ch., Haritha D., Neelima D. and Kiran kumar B.,(2008), “ Dimensionality Reduction of Covariance matrix in PCA for Face Recognition", proceesings of the International conference on Advances in Mathematics: Historical Developments and Engineering Applications (ICAM 2007), pp.400412,Utterakanda, UP.

[25] Satyanarayana Ch., Haritha D., Sammulal P. and Pratap Reddy L.,(2009), "Incremental training method for face Recognition using PCA", proceeding of the international journal of Information processing, vol. no: 3 No:1 pp. 13-23.

[26] Satyanarayana Ch., Prasad PVRD., Mallikarjuna Rao G., Haritha D., Pratap Reddy L.,(2008), “ A Comparative performance evaluation using PCA for Face Recognition", proceeding of the international journal of Science \& Technology, vol. no:4, No:4, pp. 8-16.

[27] Swets D. and Weng J. (1996), "Using discriminant eigenfeatures for image retrieval", IEEE Trans. On Patt. Anal. And Mach. Intell., 18(8), pp.831-836.

[28] Turk M. and Pentland A. (1991), "Eigenface for recognition", in Journal of Cognitive Neuro-science, 3(1), pp.70-86.

[29] Xudong Xie, Kin-Man Lam, (2006)," An efficient illumination normalization method for face recognition", Pattern Recognition Letters 27,pp.609-617.

[30] Zhang G., Huang X., Li S.Z., Wang Y., and Wu X. (2004), "Boosting local binary pattern (LBP)-based face recognition", in Proc. Advances in Biometric Person Authentication: 5th Chinese Conference on Biometric Recognition, SINOBIOMETRICS 2004 Guangzhou, China, pp.179-186. 